\title{
Partitioning tree-shaped task graphs for distributed platforms with limited memory
}

\author{
Changjiang Gou, Anne Benoit, Loris Marchal
}

\begin{abstract}
Scientific applications are commonly modeled as the processing of directed acyclic graphs of tasks, and for some of them, the graph takes the special form of a rooted tree. This tree expresses both the computational dependencies between tasks and their storage requirements. The problem of scheduling/traversing such a tree on a single processor to minimize its memory footprint has already been widely studied. The present paper considers the parallel processing of such a tree and studies how to partition it for a homogeneous multiprocessor platform, where each processor is equipped with its own memory. We formally state the problem of partitioning the tree into subtrees, such that each subtree can be processed on a single processor (i.e., it must fit in memory), and the goal is to minimize the total resulting processing time. We prove that this problem is NP-complete, and we design polynomial-time heuristics to address it. An extensive set of simulations demonstrates the usefulness of these heuristics.
\end{abstract}

Index Terms - Scheduling, tree partitioning, memory-aware, makespan minimization, parallel computing.

\section{Introduction}

$\mathrm{P}$ arallel workloads are often modeled as directed acyclic graphs of tasks. We aim at scheduling some of these graphs, namely rooted tree-shaped workflows, onto a set of homogeneous computing platforms, so as to minimize the makespan. Such tree-shaped workflows arise in several computational domains, such as the factorization of sparse matrices [?], or in computational physics code modeling electronic properties [?]. The vertices (or nodes) of the tree typically represent computation tasks, and the edges between them represent dependencies, in the form of output and input files.

In this paper, we consider out-trees, where there is a dependency from a node to each of its child nodes (the case of in-trees is similar). For such out-trees, each node (except the root) receives an input file from its parent, and it produces a set of output files (except leaf nodes), each of them being used as an input by a different child node. All its input file, execution data and output files have to be stored in local memory during its execution. The input file is discarded after execution, while output files are kept for the later execution of the children.

The way the tree is traversed influences the memory behavior: different sequences of node execution demand different amounts of memory. The potentially large size of the output files makes it crucial to find a traversal that reduces the memory requirement. In the case where even the minimum memory requirement is larger than the local memory capacity, a good way to solve the problem is to partition the tree and map the parts onto a multiprocessor computing system in which each processor has its own private memory and is

A short version of this paper was published in the proceedings of PDP'18 (Euromicro International Conference on Parallel, Distributed, and Network-Based Processing).

Manuscript received ...

Changjiang Gou is with East China Normal University, Shanghai, China. Anne Benoit, Changjiang Gou and Loris Marchal are with Univ. Lyon, CNRS, ENS de Lyon, Inria, Univ. Claude-Bernard Lyon 1, LIP UMR5668, France. Emails: \{changjiang.gou, anne.benoit, loris.marchal\}@ens-lyon.fr responsible for a single part. Partitioning makes it possible to both reduce memory requirement and to improve the processing time (or makespan) by doing some processing in parallel, but it also incurs communication costs. On modern computer architectures, the impact of communications between processors on both time and energy is non negligible, furthermore in sparse solvers it can be the bottleneck at even a small core counts [?].

The problem of scheduling a tree of tasks on a single processor to minimize the memory requirement has been studied before, and memory optimal traversals have been proposed [?], [?]. The problem of scheduling such a tree on a single processor with limited memory is also discussed in [?]: in case of memory shortage, some input files need to be moved to a secondary storage (such as a disk), which is larger but slower, and temporarily discarded from the main memory. These files will be retrieved later, when the corresponding node is scheduled. The total volume of data written to (and read from) the secondary storage is called the Input/Output volume (or I/O volume), and the objective is then to find a traversal with minimum I/O volume (MinIO problem).

In this work, we consider that the target platform is a multi-processor platform, each processor being equipped with its own memory. The platform is homogeneous, i.e., all processors have the same computing power and the same amount of memory. In case of memory shortage, rather than performing I/O operations, we send some files to another processor that will handle the processing of a part of the tree. If the tree is a linear chain, this only slows down the computation since communications need to be paid. However, if the tree is a fork graph, it is then possible to process different parts in parallel, hence potentially reducing the makespan. We propose to partition the tree into parts that are connected components, and hence each part is also a tree. The time needed to execute such a part is the sum of the time for the communication of the input file of its root and the computation time of each task in the part. The MinMakespan problem then consists in dividing the tree into parts, each part being processed by a separate processor, so that the makespan 
is minimized. The memory constraint states that we must be able to process each part within the limited memory of a single processor. This is a strict constraint, i.e., we assume that there is no secondary storage available, and the execution fails if a part cannot be executed in memory.

The main contributions of this paper are the following:

- We formalize the MinMakespan problem, and in particular we explain how to express the makespan given a decomposition of the tree into subtrees;

- We prove that MinMakespan is NP-complete;

- We design several polynomial-time heuristics aiming at obtaining efficient solutions;

- We evaluate the proposed heuristics through a set of simulations.

The paper is organized as follows. Section ?? gives an overview of related work. Then, we formalize the model in Section ??. In Section ??, we show that MinMakespan is NP-complete. All the heuristics are presented in Section ??, and the experimental evaluation is conducted in Section ?? Finally, we give some concluding remarks and hints for future work in Section??.

\section{Related work}

This study falls into the realm of scheduling tasks with precedence constraints, usually modeled as task graphs. This is a difficult scheduling problem for general graphs [?], and we focus here on the slightly simpler problem of scheduling task trees. We first review some of the existing work on scheduling such trees, especially those concerned with limiting the memory footprint. Then, we review existing work on partitioning graphs, and especially trees.

As stated above, rooted trees are commonly used to represent task dependencies for scientific applications. Liu [?] gives a detailed description of the construction of the elimination tree, its use for Cholesky and LU factorizations of sparse matrices, and its role in multifrontal methods. Scheduling trees coming from sparse linear algebra is a challenging problem because of the enormous tasks' amount and their irregular weights [?]. In [?], Liu introduces two techniques for reducing the memory requirement of post-order tree traversals. In the subsequent work [?], the post-order constraint is dropped and an efficient algorithm to find a memory-optimal schedule for task trees coming from the multifrontal method is given. Building upon Liu's work, some of us [?] proposed a new exact algorithm for scheduling a tree with the minimum memory requirement, and studied how to minimize the $\mathrm{I} / \mathrm{O}$ volume when out-of-core execution is required (MinIO problem). This work was then extended to shared-memory platforms [?], where the bi-criteria problem of minimizing makespan and memory was studied. Theoretical results were proven and heuristics were designed, but none with a strict memory constraint as in the present framework. Still, we will use and adapt the SplitSubtrees heuristic from [?] for our framework, since it splits the tree into subtrees to be processed in parallel and turns out to be quite efficient; hence it will serve as a comparison basis in this work.

Several recent studies have considered parallel sparse matrix solvers, and they have investigated techniques and algorithms to reduce communication and execution times on different systems (shared memory, distributed). Kim et al. [?] propose a two-level task parallelism algorithm, which first dynamically schedules tasks, and then further decomposes nodes into regular fine-grained tasks. In this work, the scheduling of tasks of the first level is handled by OpenMP, which however may cause an arbitrarily bad memory consumption. In a later work, Kim et al. [?] take memory bounds into consideration through Kokkos's [?] dynamic task scheduling and memory management. Agullo et al. [?] also take advantage of twolevel parallelism and discuss the ease of programming and the performance of the program. Targeting at distributed memory systems, Sao et al. [?] partition the tree into many independent subtrees and a common ancestor subtree, and then replicate the ancestor to the processors that are in charge of the children; both communication time and makespan are reduced by this method, at the expense of a larger memory consumption. Note that the subtree generated by their method may consist of many unconnected components.

Partitioning a tree (or more generally a graph) into separate subsets to optimize some metrics has been thoroughly studied (see [?] for a survey). The partition is done such that the different parts are balanced, i.e., they all have more or less the same computation weight. Most problem instances are NP-hard. When focusing on trees rather than general graphs, the balanced partitioning problem is still difficult [?]. It is APX-hard to approximate the cut size within any finite factor if subtrees are strictly balanced, some studies hence approximate the cut size as well as the balance, known as bicriteria-approximation [?]. When near-balance is allowed, tree partitioning is promising. Feldmann and Foschini [?] give a polynomial-time algorithm that cuts no more edges than an optimal perfectly balanced solution. Note that having a balanced solution is not a critical goal in our study. Indeed, each part must fit in memory, but the memory requirements of tasks of the tree do not sum up when we execute a tree: the final memory requirement of a part depends on the traversal. Hence, it would not be easy to define a relevant weight to use with classical graph partitioning approaches.

However, some recent studies use a directed acyclic graph partitioner that produces an acyclic partition of the graph, with the aim of minimizing the edge cut [?]. Building upon this partitioner, novel scheduling heuristics have been proposed to minimize the makespan [?]. Parts are scheduled using a classical list-scheduling algorithm [?], with the additional constraint that two tasks in a same part should be mapped on the same processor. Several parts may however be executed on a same processor, and the memory usage of processors is not accounted for. In a very recent paper [?], some of us have adapted the use of the partitioner to account for a memory constraint. This study focuses on a single processor and aims at minimizing the number of cache misses, in the same line as the MinIO problem mentioned earlier.

To the best of our knowledge, no study has been attempting to address the MinMakespan problem, where the execution of a tree is distributed on several processors, with one subtree per processor, a strict memory constraint, and such that the makespan is minimized.

\section{Model}

We consider a tree-shaped task graph $\tau$, where the vertices (or nodes) of the tree, numbered from 1 to $n$, correspond to 
tasks, and the edges correspond to precedence constraints among the tasks. The tree is rooted (node $r$ is the root, where $1 \leq r \leq n)$, and all precedence constraints are oriented towards the leaves of the tree. Note that we may similarly consider precedence constraints oriented towards the root by reversing all schedules, as outlined in [?]. A precedence constraint $i \rightarrow j$ means that task $j$ needs to receive a file (or data) from its parent $i$ before it can start its execution. Each task $i$ in the rooted tree is characterized by the size $f_{i}$ of its input file, and by the size $m_{i}$ of its temporary execution data (and for the root $r$, we assume that $f_{r}=0$ ). A task can be processed by a given processor only if all the task's data (input file, output files, and execution data) fit in the processor's currently available memory. More formally, let $M$ be the size of the main memory of the processor, and let $F_{i}$ be the set of files stored in this memory when the scheduler decides to execute task $i$. Note that $F_{i}$ must contain the input file of task $i$. The processing of task $i$ is possible if we have:

$$
\operatorname{MemReq}(i)=f_{i}+m_{i}+\sum_{j \in \operatorname{children}(i)} f_{j} \leq M-\sum_{j \in F_{i}, j \neq i} f_{j},
$$

where $\operatorname{MemReq}(i)$ denotes the memory requirement of task $i$, and children $(i)$ are its children nodes in the tree. Intuitively, $M$ should exceed the largest memory requirement over all tasks (denoted as MaxOutDeg in the following), so as to be able to process each task:

$$
\text { MaxOutDeg }=\max _{1 \leq i \leq n}(\operatorname{MemReq}(i)) \leq M .
$$

However, this amount of memory is in general not sufficient to process the whole tree, as input files of unprocessed tasks must be kept in memory until they are processed.

Task $i$ can be executed once its parent, denoted parent $(i)$, has completed its execution, and the execution time for task $i$ is $w_{i}$. Of course, it must fit in memory to be executed. If the whole tree fits in memory and is executed sequentially on a single processor, the execution time, or makespan, is $\sum_{i=1}^{n} w_{i}$. In this case, the task schedule, i.e., the order in which tasks of $\tau$ are processed, plays a key role in determining how much memory is needed to execute the whole tree in main memory. When tasks are scheduled sequentially, such a schedule is a topological order of the tree, also called a traversal. One can figure out the minimum memory requirement of a task tree $\tau$ and the corresponding traversal using the work of Liu [?] or some of the authors' previous work [?]. We denote by $\operatorname{MinMemory}(\tau)$ the minimum amount of memory necessary to complete task tree $\tau$.

The target platform consists of $p$ identical processors, each equipped with its own private memory of size $M$. The aim is to benefit from this parallel platform both for memory, by allowing the execution of a tree that does not fit within the memory of a single processor, and also for makespan, since several parts of the tree could then be executed in parallel. The goal is therefore to partition the tree workflow $\tau$ into $k \leq p$ parts $\tau_{1}, \ldots, \tau_{k}$, which are connected components of the original tree. Hence, each part $\tau_{i}$ is itself a tree. We refer to these connected components as subtrees of $\tau$. Note that $\tau$ can also be viewed as a tree made of these subtrees. Such a partition is illustrated in Figure ??, where the tree is decomposed into five subtrees: $\tau_{1}$ with nodes 1 , 2 , and $3 ; \tau_{2}$ with nodes 4,6 , and $7 ; \tau_{3}$ with node $5 ; \tau_{4}$

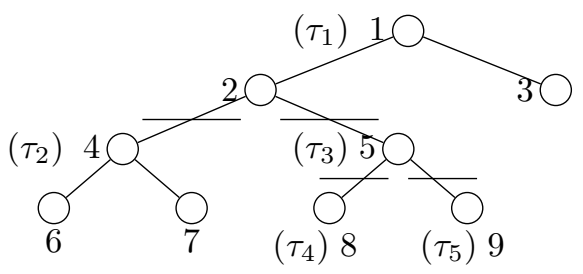

Figure 1: Partition and recursive computation of makespan.

with node 8 ; and $\tau_{5}$ with node 9 . We require that each subtree $\tau_{i}$ can be each executed within the memory of a single processor (no secondary storage such as disk is available), i.e., $\operatorname{MinMemory}\left(\tau_{\ell}\right) \leq M$, for $1 \leq \ell \leq k$.

We are to execute such $k$ subtrees on $k$ processors. Let $\operatorname{root}\left(\tau_{\ell}\right)$ be the task at the root of subtree $\tau_{\ell}$. If $\operatorname{root}\left(\tau_{\ell}\right) \neq r$, the processor in charge of tree $\tau_{\ell}$ needs to receive some data from the processor in charge of the tree containing parent $\left(\operatorname{root}\left(\tau_{\ell}\right)\right)$, and this data is a file of size $f_{\operatorname{root}\left(\tau_{\ell}\right)}$. This can be done within a time $\frac{f_{\operatorname{root}\left(\tau_{\ell}\right)}}{\beta}$, where $\beta$ is the available bandwidth between each couple of processors.

We denote by alloc $(i)$ the set of tasks included in subtree $\tau_{\ell}$ rooted in $\operatorname{root}\left(\tau_{\ell}\right)=i$, and by $\operatorname{desc}(i)$ the set of tasks, not in $\operatorname{alloc}(i)$, that have a parent in alloc $(i)$ :

$$
\operatorname{desc}(i)=\{j \notin \operatorname{alloc}(i) \mid \operatorname{parent}(j) \in \operatorname{alloc}(i)\} .
$$

The makespan can then be expressed with a recursive formula. Let $M S(i)$ denote the time (or makespan) required to execute the whole subtree rooted in $i$, given a partition into subtrees. Note that the whole subtree rooted in $i$ may contain several subtrees of the partition (it is $\tau$ for $i=r$ ). The goal is hence to express $M S(r)$, which is the makespan of $\tau$. We have (recall that $f_{r}=0$ by convention):

$$
M S(i)=\frac{f_{i}}{\beta}+\sum_{j \in \operatorname{alloc}(i)} w_{j}+\max _{k \in \operatorname{desc}(i)} M S(k) .
$$

We assume that the whole subtree $\tau_{\ell}$ is computed before initiating communication with its children.

The goal is to find a decomposition of the tree into $k \leq p$ subtrees that all fit in the available memory of a processor, so as to minimize the makespan $M S(r)$. Figure ?? exhibits an example of such a tree decomposition, where the horizontal lines represent the edges cut to disconnect the tree $\tau$ into five subtrees. Subtree $\tau_{1}$ is executed first, after receiving its input file of size $f_{1}=0$, and it includes tasks 1,2 and 3 . Then, subtrees $\tau_{2}$ and $\tau_{3}$ are processed in parallel. The final makespan for $\tau_{1}$ is thus:

$$
M S(1)=\frac{f_{1}}{\beta}+w_{1}+w_{2}+w_{3}+\max (M S(4), M S(5)),
$$

where $M S(5)$ recursively calls $\max (M S(8), M S(9))$, since $\tau_{4}$ and $\tau_{5}$ can also be processed in parallel.

For convenience, we also denote by $W_{i}$ the sum of the weights of all nodes in the subtree rooted in $i$ (hence, for a leaf node, $\left.W_{i}=w_{i}\right)$ :

$$
W_{i}=w_{i}+\sum_{j \in \operatorname{children}(i)} W_{j} .
$$

We are now ready to formalize the optimization problem that we consider: 
Definition 1 (MinMakespan). Given a task tree $\tau$ with $n$ nodes, a set of $p$ processors each with a fixed amount of memory $M$, partition the tree into $k \leq p$ subtrees $\tau_{1}, \ldots, \tau_{k}$ such that $\operatorname{MinMemory}\left(\tau_{i}\right) \leq M$ for $1 \leq i \leq k$, and the makespan is minimized.

Given a tree $\tau$ and its partition into subtrees $\left\{\tau_{1}, \ldots, \tau_{k}\right\}$, we consider its quotient graph $Q$ given by the partition: vertices from a same subtree are represented by a single vertex in the quotient tree, and there is an edge between two vertices $u \rightarrow v$ of the quotient graph if and only if there is an edge in the tree between two vertices $i \rightarrow j$ such that $i \in \tau_{u}$ and $j \in \tau_{v}$. Note that since we impose a partition into subtrees, the quotient graph is indeed a tree. This quotient tree will be helpful to compute the makespan and to exhibit the dependencies between the subtrees.

\section{Problem complexity}

Theorem 1. The (decision version of) MinMakespan problem is NP-complete.

Proof. First, it is easy to check that the problem belongs to NP: given a partition of the tree into $k \leq p$ subtrees, we can check in polynomial time that (i) the memory needed for each subtree does not exceed $M$, and that (ii) the obtained makespan is not larger than a given bound.

To prove the completeness, we use a reduction from 2partition [?]. We consider an instance $\mathcal{I}_{1}$ of 2-partition: given $n$ positive integers $a_{1}, \ldots, a_{n}$, does there exist a subset $I$ of $\{1, \ldots, n\}$ such that $\sum_{i \in I} a_{i}=\sum_{i \notin I} a_{i}=S / 2$, where $S=\sum_{i=1}^{n} a_{i}$. We consider the 2-partition-equal variant of the problem, also NP-complete, where both partitions have the same number of elements $(|I|=n / 2$, and thus, $n$ is even). Furthermore, we assume that $n \geq 4$, since the problem is trivial for $n=2$. From $\mathcal{I}_{1}$, we build an instance $\mathcal{I}_{2}$ of MinMakespan as follows:

- The tree $\tau$ consists of $n+2$ nodes, and it is described in Figure ??: it is a fork graph (a root with $n+1$ children). The weights on edges represent the size of input files $f_{i}$, and the computation time and memory requirements are indicated respectively by $w_{i}$ and $m_{i}(0 \leq i \leq n+1$, where root $=0)$.

- For $1 \leq i \leq n, w_{i}=S-a_{i}, m_{i}=M-\sum_{j=1}^{i} a_{j}$, and $f_{i}=a_{i}$.

- For the last child, $w_{n+1}=0, m_{n+1}=S / 2$, and $f_{n+1}=$ $M-S$.

- For the root, $w_{\text {root }}=0, m_{\text {root }}=0$, and $f_{\text {root }}=0$.

- The makespan bound is $C_{\max }=(n+1) \frac{S}{2}$.

- The memory bound is $M=C_{\max }+S+1$.

- The bandwidth is $\beta=1$.

- The number of processors is $p=\frac{n}{2}+1$.

Consider first that $\mathcal{I}_{1}$ has a solution, $I$, such that $I \subseteq\{1, \ldots, n\}$ and $|I|=n / 2$ (i.e., $I$ contains exactly $n / 2$ elements). We execute sequentially root and task $n+1$, plus the tasks in $I$, and we pay communications and execute in parallel tasks not in $I$. We can execute each of these tasks in parallel since there are $n / 2+1$ processors and exactly $n / 2$ tasks not in $I$. Since we have cut nodes not in $I$, there remains exactly files of size $S / 2$ in memory, plus $f_{2 n+1}=M-S$,

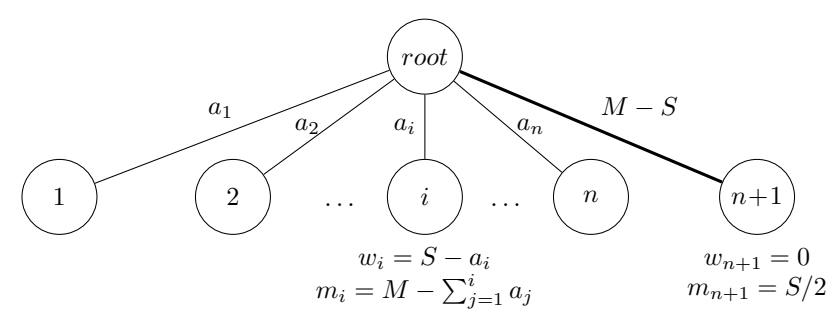

Figure 2: Tree of instance $\mathcal{I}_{2}$ used in the NPC proof.

and to execute task $n+1$, we also need to accommodate $m_{2 n+1}=S / 2$, hence we use exactly a memory of size $M$. We can then execute nodes in $I$ starting from the right of the tree, without exceeding the memory bound. Indeed, once task $n+1$ has been executed, there remains only some of the $f_{i}=a_{i}$ 's in memory, and they fit together with $m_{i}$ in memory. The makespan is therefore $\frac{n}{2} S-\frac{S}{2}$ for the sequential part (executing all tasks in $I$ ), and each of the tasks not in $I$ can be executed within a time $S$ (since $\beta=1$ ), all of them in parallel, hence a total makespan of $(n-1) \frac{S}{2}+S=C_{\max }$. Hence, $\mathcal{I}_{2}$ has a solution.

Consider now that $\mathcal{I}_{2}$ has a solution. First, because of the constraint on the makespan, root and task $n+1$ must be in the same subtree, otherwise we would pay a communication of $M-S=C_{\max }+1$, which is not acceptable. Let $I$ be the set of tasks that are executed on the same subtree as root and task $n+1$. $I$ contains at least $\frac{n}{2}$ tasks, since the number of processors is $\frac{n}{2}+1$. If $I$ contains more than $\frac{n}{2}$ tasks, then the makespan is strictly greater than $\left(\frac{n}{2}+1\right) S-S$ for the sequential part, plus $S$ for all other tasks done in parallel, that is $\left(\frac{n}{2}+1\right) S>C_{\max }$. Therefore, $I$ contains exactly $\frac{n}{2}$ tasks.

The constraint on makespan requires that $\frac{n}{2} S-\sum_{i \in I} a_{i}+$ $S \leq C_{\max }$, and hence $\sum_{i \in I} a_{i} \geq \frac{S}{2}$. After executing root, the files remaining in memory are the files from tasks in $I$ and $f_{n+1}$, since other files are communicated to other processors. As long as $f_{n+1}$ is in memory, no task of $I$ can be executed due to the memory constraint, hence to execute task $n+1$, the memory constraint writes $\sum_{i \in I} a_{i}+M-S+\frac{S}{2} \leq M$, hence $\sum_{i \in I} a_{i} \leq \frac{S}{2}$. Therefore, we must have $\sum_{i \in I} a_{i}=\frac{S}{2}$, and we have a solution to $\mathcal{I}_{1}$.

\section{Heuristic strategies}

In this section, we design polynomial-time heuristics to solve the MinMakespan problem. The heuristics work in three steps: (1) partition the tree into subtrees in order to minimize the makespan, without accounting for the memory constraint; (2) partition subtrees that do not fit in memory, i.e., such that $\operatorname{MinMemory}\left(\tau_{i}\right)>M ;(3)$ ensure that we do have the correct number of subtrees, i.e., merge some subtrees if there are more subtrees than processors, or further split subtrees if there are extra processors and the makespan can be reduced. We now detail the three steps, focusing on makespan, then memory, then number of processors.

\subsection{Step 1: Minimizing the makespan}

In the first step, the objective is to split the tree into a number of subtrees, each processed by a single processor, in order to minimize the makespan. We will consider the memory constraint on each subtree at the next step (Section ??).

We first consider the case where the tree is a linear chain, and prove that its optimal solution uses a single processor. 
Lemma 1. Given a tree $\tau$ such that all nodes have at most one child (i.e., it is a linear chain), the optimal makespan is obtained by executing $\tau$ on a single processor, and the optimal makespan is $\sum_{i=1}^{n} w_{i}$.

Proof. If more than one processor is used, all tasks are still executed sequentially because of dependencies, but we further need to account for communicating the $f_{i}$ 's between processors. Therefore, the makespan can only be increased.

More generally, if the decomposition into subtrees form a linear chain, as defined below, then the subtrees must be executed one after the other, no parallelism is exploited and unnecessary communication may occur.

Definition 2 (Chain of subtrees). Given a tree $\tau$, its partition into subtrees $\tau_{i}$ and the resulting quotient tree $Q$, a chain of subtrees is a set of nodes $u_{1}, \ldots u_{k}$ of $Q$ such that $u_{i}$ is the only child of $u_{i-1}(i>1)$.

Therefore, having several subtrees as a linear chain can only increase the makespan, compared to an execution of the whole tree on a single processor.

We now propose three heuristics that aim at minimizing the makespan, and hence avoid having chains of subtrees.

\subsubsection{Two-level heuristic}

The first heuristic, SplitSubtrees, is adapted from [?], where the goal was to reduce the makespan while limiting the memory in a shared-memory environment. It creates a twolevel partition with one subtree containing the root, executed first on a single processor (and called the sequential set), followed by the parallel processing of $p-1$ independent subtrees. In the context of shared memory, this heuristic has been proven the two-level partition with best makespan [?, Lemma 5.1]. We adapt it to our context, in order to take communications into account.

The SplitSubtrees heuristic relies on a splitting algorithm, which maintains a set of subtrees and iteratively splits the subtree with the largest makespan. Initially, the only subtree is the whole tree. When a subtree is split, its root is moved to the sequential set (denoted seqSet) and all its children subtrees are added to the current set of subtrees. Algorithm ?? formalizes the heuristic, in which the current set of subtrees is stored in a priority queue $P Q$ sorted by nonincreasing makespan, computed using Equation (??). Note that SplitSubtrees is defined on a subtree $T \subseteq \tau$, however the makespan is always computed considering the global tree and its decomposition, which are considered as a common knowledge of all algorithms (to avoid adding parameters to the algorithms). SplitSubtrees is called on the whole tree $(T=\tau)$ for this heuristic.

For a given state of the algorithm (i.e., a partition of the tree between seqSet and subtrees in $P Q$ ), we consider the following mapping: the $p-1$ largest subtrees in $P Q$ (in terms of total computation weight $W$ ) are allocated to distinct processors, while the remaining subtrees are processed by the same processor in charge of the sequential set. Note that all these nodes ( seqSet plus the smallest subtrees of $P Q$ ) form a subtree of the original tree: seqSet is a subtree containing the root, and each root of a subtree in $P Q$ has its parent in seqSet.

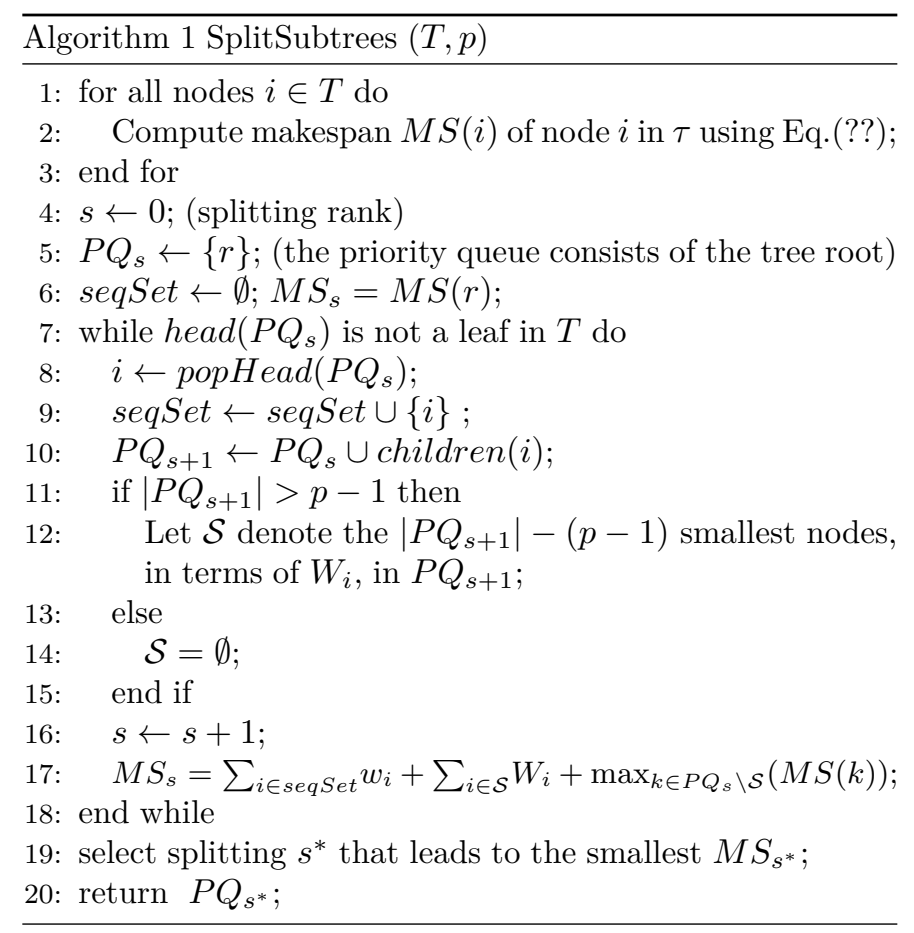

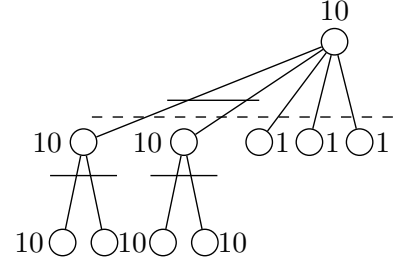

(a)

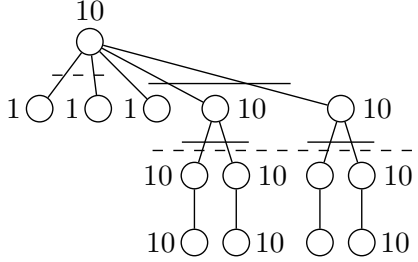

(b)
Figure 3: Two cases where SplitSubtrees is suboptimal. Dashed edges represent the solution of SplitSubtrees, plain edges give the optimal partition.

We iteratively consider the solutions obtained by the successive splitting operations and finally select the one with the best makespan. We stop splitting subtrees when the largest subtree in $P Q$ is indeed a leaf. Thus, there are at most $n$ iterations. At each iteration, the insertion into $P Q$ costs $O(\log n)$, and computing the max at Line ?? costs $O(p)$, hence the complexity is $O(n \times(\log n+p))$. It is therefore a polynomial-time algorithm. The algorithm returns the set of nodes that are the root of a subtree, which corresponds to a cut of the tree, i.e., the set of edges that are cut to partition the tree into subtrees.

\subsubsection{Improving the SplitSubtrees heuristic}

There are two main limitations of SplitSubtrees. First, it produces only a two-level solution: in the provided decomposition, all subtrees except one are the children of the subtree containing the root. In some cases, as illustrated in Figure ??, it is beneficial to split the tree into more levels. In these examples, we have $p=7$ processors. Node labels denote their computational weights (10 for all nodes, except three of them per tree), and there are no communication costs. The horizontal dashed lines represent the edges cut in the solution of SplitSubtrees, while solid lines represent the optimal partition. In the example of Figure ??(a), a two-level solution cannot achieve a makespan better than 40 . If the 
cut was made at a lower level, the makespan would be even greater. It is however possible to achieve a makespan of 33 by cutting at two levels.

The second limitation is the possibly too large size of the first subtree, containing the sequential set seqSet. Since its execution is sequential, it may lead to a large resource waste. This is for instance the case in the example of Figure ??(b), where the optimal two-level solution has a sequential set whose execution time is 31 , while further parallelism could have been used: the optimal solution cuts this sequential set in order to minimize the makespan.

To address these limitations, we design a new heuristic, ImprovedSplit (see Algorithm ??), which improves upon SplitSubtrees by building a multi-level solution. Since we aim at further cutting the tree to obtain a multi-level solution, ImprovedSplit does not set a limit on the number of subtrees in a first step, but rather tries to create as many subtrees as possible, while the makespan can be improved. It is initially called with $T=\tau$, and first calls SplitSubtrees with no restriction on the number of subtrees: $p$ is set to $+\infty$. Then, ImprovedSplit recursively tries to split the sequential set and the largest children subtrees (subtrees whose roots are in $P Q$ ), until the makespan cannot be further reduced (again, with no restriction on the number of subtrees).

Finally, once all splits have been done, if there are more subtrees than processors, some of them are merged with a call to Merge (which will be explained in Section ??), without accounting for the memory constraint (call with infinite memory). The use of AlreadyOptSet ensures that ImprovedSplit is called at most once on each node. The makespan computation in the repeat loop has a complexity in $O(n)$, and the loop has at most $n$ iterations. Therefore, we get a complexity in $O\left(n^{2}\right)$ for a call to ImprovedSplit, without the final call to Merge, hence a complexity in $O\left(n^{3}\right)$ for the $n$ calls. Note that Merge does not do anything when $p=+\infty$, since there are enough processors, and during each recursive call to ImprovedSplit, Merge is called with $p=+\infty$, hence has no effect. The complexity of the final Merge is in $O\left(n^{3}\right)$, as we do not consider the memory constraint (see Section ??). The final complexity of ImprovedSplit is thus $O\left(n^{3}\right)$.

\subsubsection{ASAP heuristic}

The main idea of this heuristic is to parallelize the processing of tree $\tau$ as soon as possible, by cutting edges that are close to the root of the tree. ASAP uses a node priority queue $P Q$ to store all the roots of subtrees produced. Nodes in $P Q$ are sorted by non-increasing $W_{i}$ 's (recall that $W_{i}$ is the total computation weight of the subtree rooted at node $i$ ). Iteratively, the heuristic cuts the largest subtree, if it has siblings, until there are as many subtrees as processors (see Algorithm ?? for details). Therefore, it creates a multi-level partition of the tree. It selects the partition that has the minimum makespan.

At this point, we might have chains of subtrees (as defined above), which increases the makespan compared to a sequential execution of these subtrees. Figure ?? provides an example where this happens: the makespan is $11+2+12+2+10+(2+10)=49$, since the three leaf tasks of weight 10 are executed in parallel. Four units of communication time could however be saved by executing all other nodes
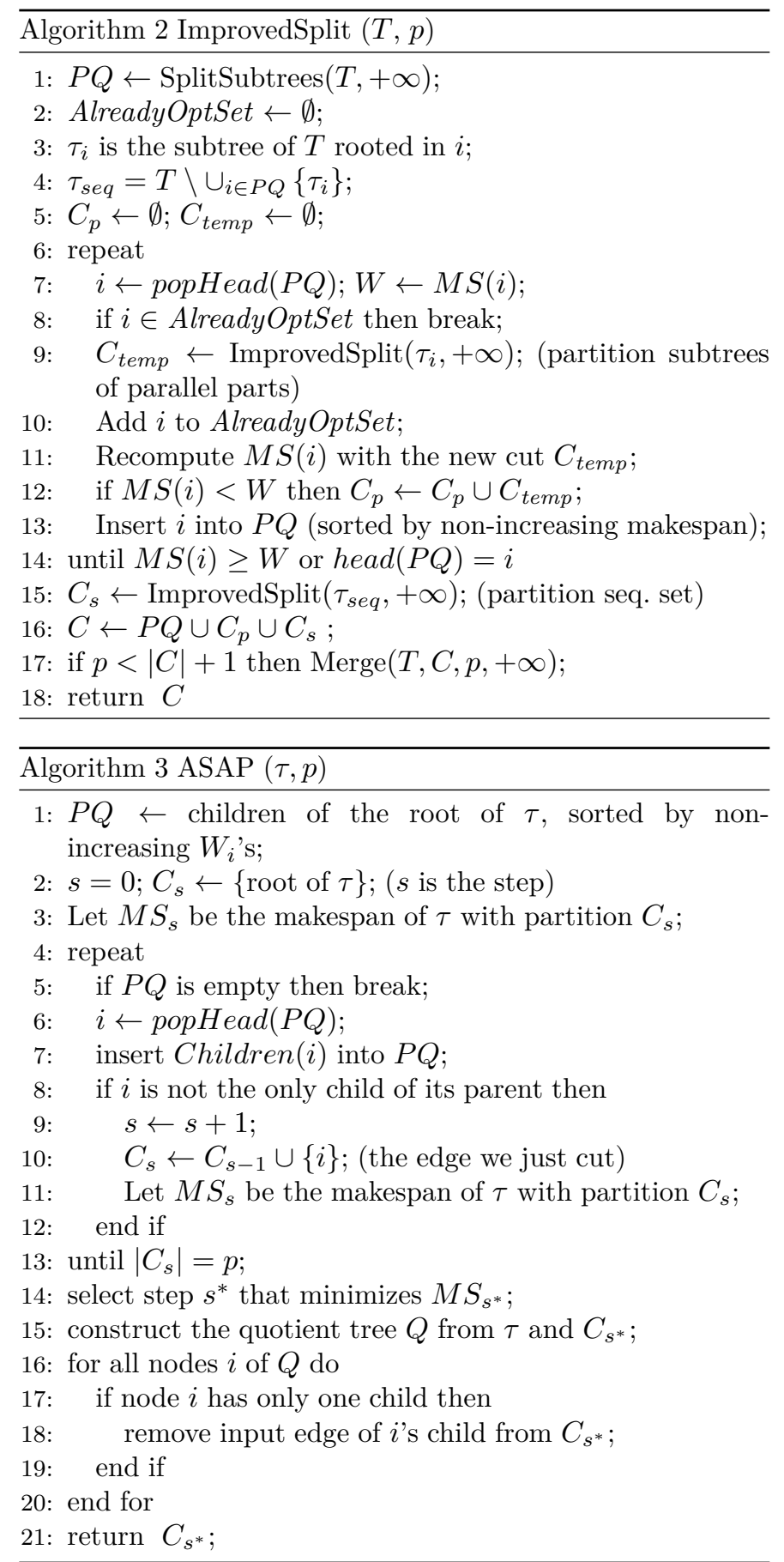

on the same processor, reaching a makespan of 45 and using only four processors.

To avoid this shortcoming, ASAP then builds the quotient tree in which, except the root, other nodes that have no siblings are elements of chains. Their input edges are therefore restored, i.e., subtrees are merged into a single subtree so that there are no more chains, and therefore, this leaves some processors idle. These idle processors will be used, if possible, to improve the makespan, during the last step of the heuristics, see Section ??.

\subsection{Step 2: Fitting into memory}

After partitioning a tree into many subtrees by SplitSubtrees, ImprovedSplit or ASAP, we propose three heuristics in this 


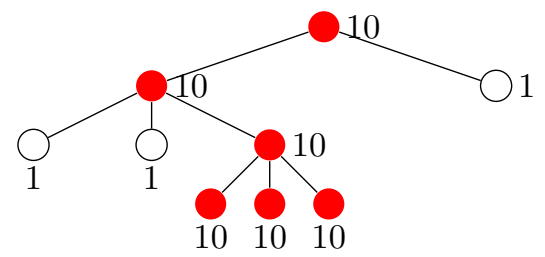

Figure 4: Example with a chain. Node labels represent their weight. All edges have weight 2 , and $p=6$. Red nodes denote subtrees' roots as determined by ASAP.

section to check each subtree's minimum memory requirement and further partition those such that $\operatorname{MinMemory}\left(\tau_{i}\right)>M$.

\subsubsection{FirstFit heuristic}

We first note the proximity of this problem with the MinIO problem [?]. In this problem, a similar tree has to be executed on a single processor with limited memory. When the memory shortage happens, some data have to be evicted from the main memory and written to disk. The goal is to minimize the total volume of the evicted data while processing the whole tree. In [?], six heuristics are designed to decide which files should be evicted. In the corresponding simulations, the FirstFit heuristic demonstrated better results. It first computes the traversal (permutation $\sigma$ of the nodes that specifies their execution sequence) that minimizes the peak memory, using the provided MinMem algorithm [?]. Given this traversal, if the next node to be processed, denoted as $j$, is not executable due to memory shortage, we have to evict some data from the memory to the disk. The amount of freed memory should be at least $\operatorname{Need}(i)=\left(\operatorname{MemReq}(j)-f_{j}\right)-M^{\text {avail }}$, where $M^{\text {avail }}$ is the currently available memory when we try to execute node $j$. In that case, FirstFit orders the set $I=\left\{f_{i_{1}}, f_{i_{2}}, \ldots, f_{i_{j}}\right\}$ of the data already produced and still residing in main memory, so that $\sigma\left(i_{1}\right)>\sigma\left(i_{2}\right)>\cdots>\sigma\left(i_{j}\right)$, where $\sigma(i)$ is the step of processing node $i$ in the traversal $\left(f_{i_{1}}\right.$ is the data that will be used for processing the latest) and selects the first data from $I$ until their total size exceeds or equals $N e e d(j)$.

We consider the simple adaptation of FirstFit to our problem: the final set of data $F$ that are evicted from the memory defines the edges that are cut in the partition of the tree, thus resulting in $|F|+1$ subtrees. This guarantees that each subtree can be processed without exceeding the available memory, but may lead to numerous subtrees.

\subsubsection{LargestFirst heuristic}

For our problem, we want to end up with a total of not more than $p$ subtrees from the original tree (one subtree per processor), and since we may have already created $p$ subtrees in Step 1 (Section ??), we do not want to create too many additional subtrees. Otherwise, subtrees will have to be merged in Step 3 (Section ??), possibly resulting in an increase of makespan. Therefore, we propose a variant of the FirstFit strategy, which orders the set $I$ of candidate data to be evicted by non-increasing sizes $f_{i}$, and selects the largest data until their total size exceeds the required amount. This may result into edges with larger weights being cut, and thus an increased communication time, but it is likely to reduce the number of subtrees. This heuristic is called LargestFirst.

\subsubsection{Immediately heuristic}

The third heuristic for Step 2, Immediately, also starts from a minimum memory sequential traversal $\sigma$. We simulate the execution of $\sigma$, and each time we encounter a node that is not executable because of memory shortage, we cut the corresponding edge and this node becomes the root of a new subtree. We continue the process for the remaining nodes, and then recursively apply the same procedure on all created subtrees, until each of them fits in memory.

\subsection{Step 3: Reaching an acceptable number of subtrees}

Now that we have first minimized the makespan, and then made sure that each subtree fits in local memory, we need to check how many subtrees have been generated. During this step, we either decrease the number of subtrees if it is greater than the number of processors $p$, or we increase it by further splitting subtrees if we have idle processors and the makespan may be improved.

\subsubsection{Decreasing the number of subtrees}

If there are more subtrees than processors, some of them have to be merged, and the resulted subtrees should also fit in local memory.

For subtrees that are leaves and have only one sibling, merging only theirselves to their parents will lead to a chain, which wastes processors. Thus, they are also merged with their siblings. In all combinations that fit in memory, we greedily merge subtrees that lead to the minimum increase in makespan. We compute the increase in makespan as follows. We denote the subtree to be merged as node $i$ of the quotient tree. Sometimes (when $i$ is not on the critical path), $M S(i)$ can be increased without changing the final makespan $M S(r)$. We define $d_{i}$ as the slack in $M S(i)$, that is, the threshold such that $M S(r)$ is not impacted by the increase of $M S(i)$ up to $M S(i)+d_{i}$. It can be recursively computed from the root: $d_{i}=d_{t}+M S(k)-M S(i)$, in which $t$ is $i$ 's parent in the quotient tree and $k$ is the sibling of $i$ that has the maximum makespan. For the root, $d_{r}$ is set to 0 .

We then compute the increase of makespan of merging $i$ to its parent $t$ in the quotient tree as follows. We first estimate the increase $\Delta_{t}$ of $M S(t)$. If $i$ is a leaf and has only one sibling, denoted $j$, the increase in makespan of their parent $t$ is $\Delta_{t}=W_{i}+W_{j}-\max \left(\frac{f_{i}}{\beta}+w_{i}, \frac{f_{j}}{\beta}+w_{j}\right)$. For other subtrees, the makespan of $t$ before the merge is $M S(t)=\frac{f_{t}}{\beta}+W_{t}+\max (M S(k), M S(i))$, and after merging $i$ to $t, M S(t)=\frac{f_{t}}{\beta}+W_{t}+W_{i}+\max (M S(k), M S(j))$, where $j$ is the child of $i$ that has the maximum makespan. Therefore, the increase of $M S(t)$ is $\Delta_{t}=W_{i}+\max (M S(k), M S(j))-$ $\max (M S(k), M S(i))$. Finally, taking the slack into consideration, the increase of $M S(r)$ is $\Delta_{t}-d_{i}$.

This algorithm is formalized as Algorithm ??, where shortage represents the number of subtrees that should be merged. There are initially at most $n$ subtrees. For each possible combination, computing the $\Delta$ increase costs $O(n)$, and computing the minimum memory consumption costs $O(n \log n)$. There are at most $n$ subtrees in $Q$, and at least one subtree is removed at each iteration, hence the complexity is $O\left(n^{3} \log n\right)$. Note that when the memory provided to the Merge algorithm is unbounded $(M=+\infty)$, the tests to check if a subtree fits in memory $M$ may be skipped, which reduces the complexity of Merge to $O\left(n^{3}\right)$. 


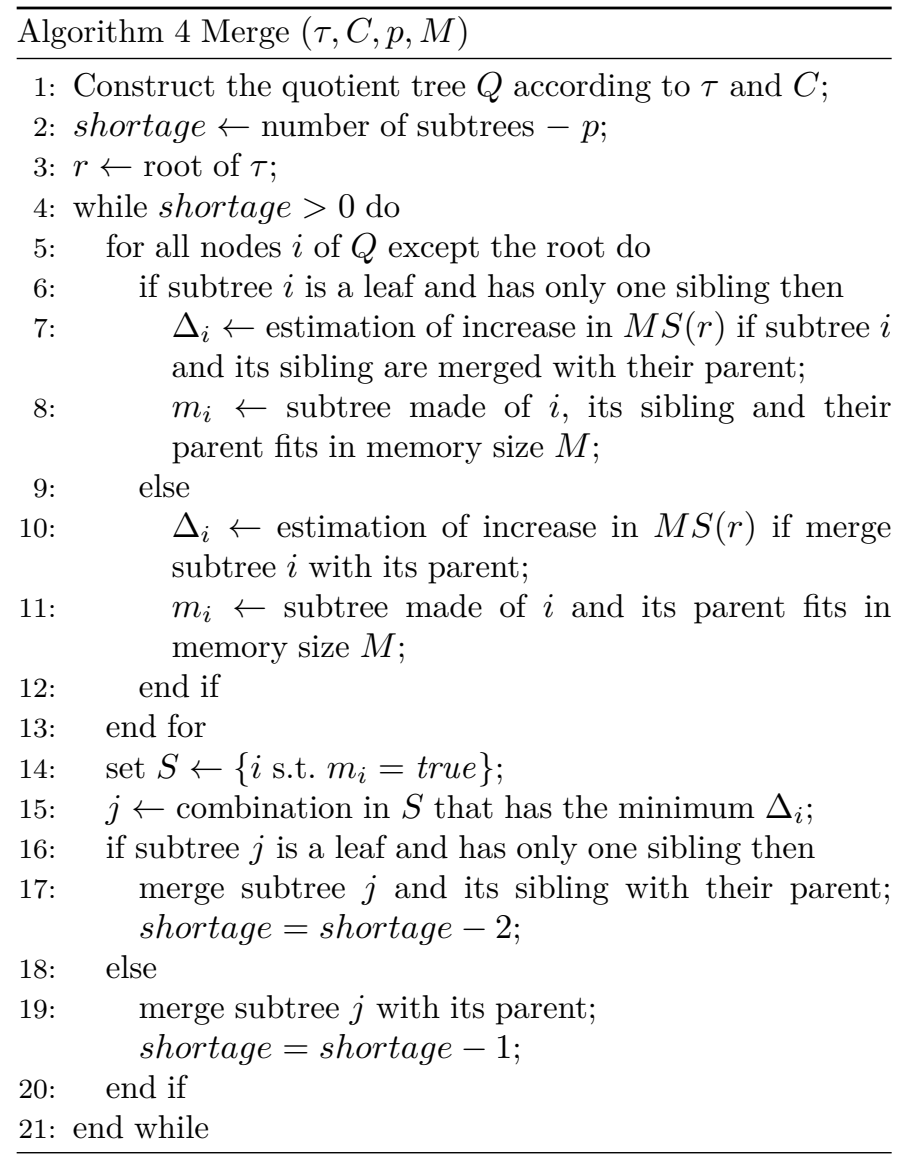

\subsubsection{Increasing the number of subtrees}

If there are more processors than subtrees, we may be able to further reduce the makespan by splitting some of the subtrees. Given a tree $\tau$ and a partition $C$, SplitAgain first builds the quotient tree $Q$ to model dependencies among subtrees, and finds its critical path. A critical path is a set of nodes of $Q$ that defines the makespan of $\tau$. In the example of Figure ??, the critical path consists of three nodes of the quotient tree. Each subtree on the critical path is a candidate to be cut into two (or three) parts by cutting some edges. The set $L$ (black nodes in Figure ??) contains the nodes whose input edge could be cut. If the subtree is a leaf in the quotient tree, we always split into three parts, otherwise we would create a chain and only increase the makespan. At each step, we greedily select the option (within nodes of $L$ ) that has the maximum potential decrease in makespan of $\tau$. We compute the potential makespan decrease as follows: let $i$ be the node whose input edge is considered to be cut. It currently lies in the subtree $\tau_{t}$ rooted at node $t$. After cutting the input edge of node $i$, it produces a new subtree $\tau_{i}$ of weight $W_{i}$.

The makespan of $\tau_{t}$ after cutting is given by:

$$
\max \left(M S(t)-W_{i}, W_{t}+\frac{f_{i}}{\beta}+\max _{\tau_{j} \in \operatorname{Children}\left(\tau_{i}\right)} M S(j)\right),
$$

where $M S(j)$ is the makespan of a subtree rooted at $j$ before cutting any new edge. Indeed, either the critical path does not include $\tau_{i}$, or it now includes the communication to $\tau_{i}$ and the makespan of the largest children of $\tau_{i}$ in the new quotient tree. Note that if the child of $\tau_{t}$ that is in the critical path is also a child of $\tau_{i}$ (for instance, in Figure ??, when we
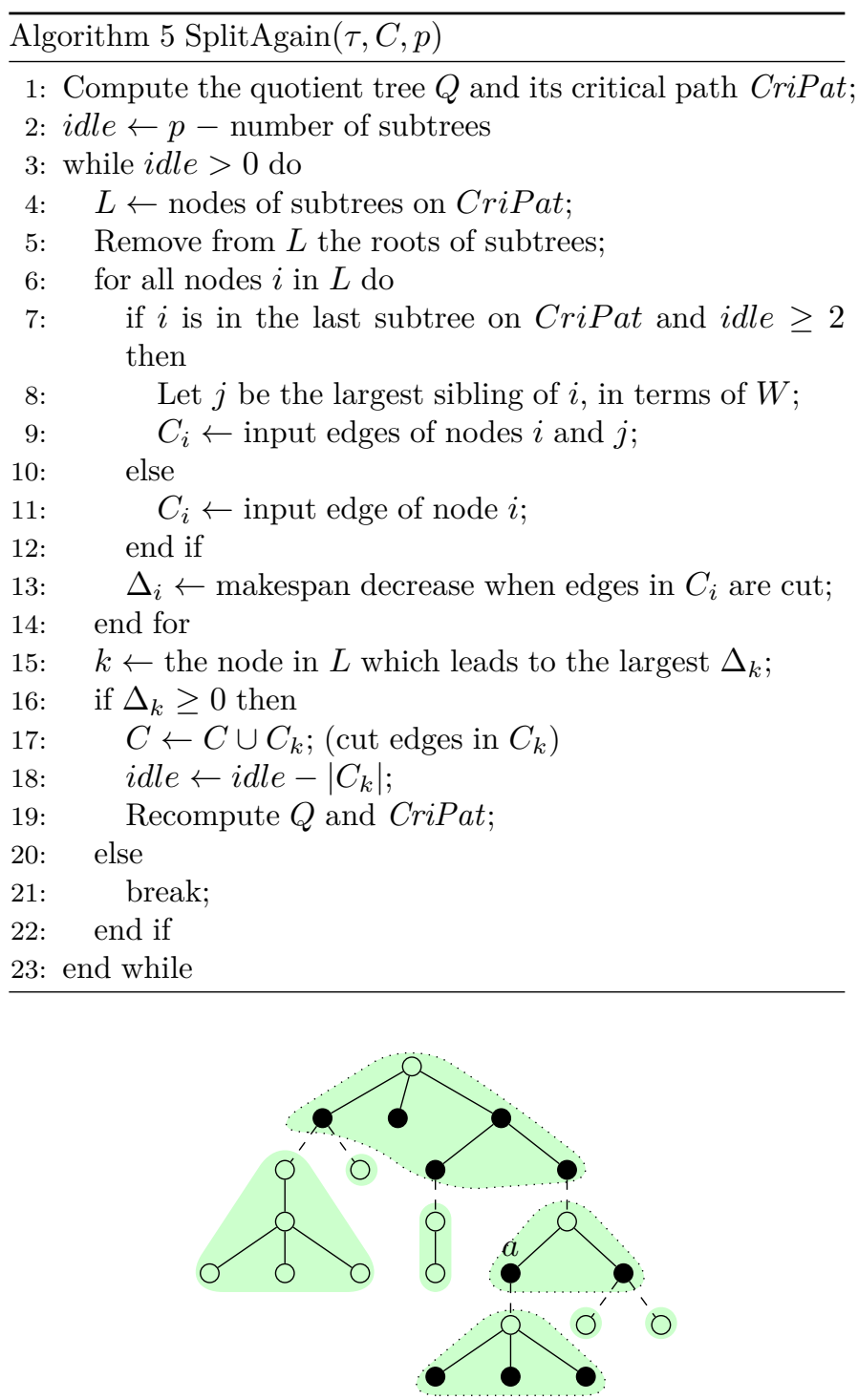

Figure 5: Example to illustrate SplitAgain: green areas surrounded with dotted line belong to the critical path; black nodes are candidates to be cut after line ?? (set $L$ ).

try to cut the input edge of node $a$ ), the makespan will only be increased, and hence we will never cut edge $i$.

The decrease of the makespan of $\tau_{t}$ when cutting the input edge of node $i$ is thus given by:

$$
\Delta_{i}=\min \left(W_{i}, M S(t)-W_{t}-\frac{f_{i}}{\beta}-\max _{\tau_{j} \in \operatorname{Children}\left(\tau_{i}\right)} M S(j)\right) .
$$

If we cut two edges in the last subtree on the critical path, say $i$ and $j$, the makespan after cutting is

$$
M S(t)-W_{i}-W_{j}+\max \left(\frac{f_{i}}{\beta}+W_{i}, \frac{f_{j}}{\beta}+W_{j}\right),
$$

and the decrease of $M S(t)$ is:

$$
\Delta_{i}=\min \left(W_{j}-\frac{f_{i}}{\beta}, W_{i}-\frac{f_{j}}{\beta}\right) .
$$

This process is repeated until there are no more idle processors or no further decrease in makespan. It is formalized in Algorithm ??. There are at most $p$ processors left to use. At each iteration, $L$ has at most $n$ nodes, and computing $\Delta_{i}$ costs $O(n)$, hence the complexity is $O\left(p n^{2}\right)$. 


\section{Experimental validation through simulations}

In this section, we compare the performance of the proposed heuristics on a wide range of computing platform settings. We evaluate the results of the three steps: partition for reducing makespan, fitting in the memory constraint, and constraint on the number of processors.

\subsection{Dataset and simulation setup}

The dataset contains assembly trees of a set of sparse matrices obtained from the University of Florida Sparse Matrix Collection. We selected square matrices, whose number of rows is between $2 \times 10^{4}$ and $10^{6}$, and whose number of non-zeros per row is at least 2.5, and the total number of non-zeros is at most $5 \times 10^{6}$. These 76 matrices were first ordered using AMD or MeTiS, then the corresponding elimination trees were built, and relaxed node amalgamation was performed on these trees (see [?] for more details on this construction).

To test the heuristics proposed above, we only kept trees whose MinMemory is larger than its MaxOutDeg. This corresponds to 31 trees in the data set, coming from 22 matrices.

To compare the performance of the proposed heuristics in different environments, and since these trees exhibit very different number of nodes, we have selected three different processor to node ratios (PNR): the number of processors $p$ can be set to $1 e-04,0.001$, or 0.01 times the tree size $n$ (while ensuring $p \geq 3$ ). We also consider three scenarios for the relative cost of computations vs. communications. Given a tree, we select the communication bandwidth $\beta$ such that the average communication to computation ratio (CCR), defined as the total time for communicating all data divided by the total computation time, is either $0.1,1$ or 10 .

We consider two scenarios for the memory constraint: (i) in the loose scenario, the memory bound for each processor is set to MinMemory, hence there is no memory constraint; (ii) then, the strict scenario sets the memory bound to MaxOutDeg, the minimum memory needed to process any single task. The sequential tree traversal used in FirstFit, LargestFirst and Immediately is given by MinMem as described in [?], which has a minimum memory cost. All algorithms are implemented in $\mathrm{C}++$, compiled by $\mathrm{g}++6.3 .0$, and executed on a platform based on Intel Xeon E5520 processors and Linux Debian 4.9.168-1. All codes and trees can be found at https://github.com/gouchangjiang/MemComJournal and on https://codeocean.com for reproducibility purpose.

\subsection{Step 1: Minimizing the makespan}

The results of heuristics for reducing makespan on different computing scenarios are shown in Figure ??. We consider all combinations of CCRs and PNRs, and we normalize the makespan of SplitSubtrees, ImprovedSplit and ASAP to the makespan obtained with a sequential execution of the tree, denoted by Sequence. Hence, a smaller ratio indicates a better relative performance. Note that there is no memory constraint in this step, hence Sequence returns a valid solution, using only one processor.

As expected, all heuristics achieve significant gain compared to the reference sequential schedule Sequence: the makespan is reduced by at least $45 \%$ on more than $50 \%$ of the cases. With more processors, they behave even better than
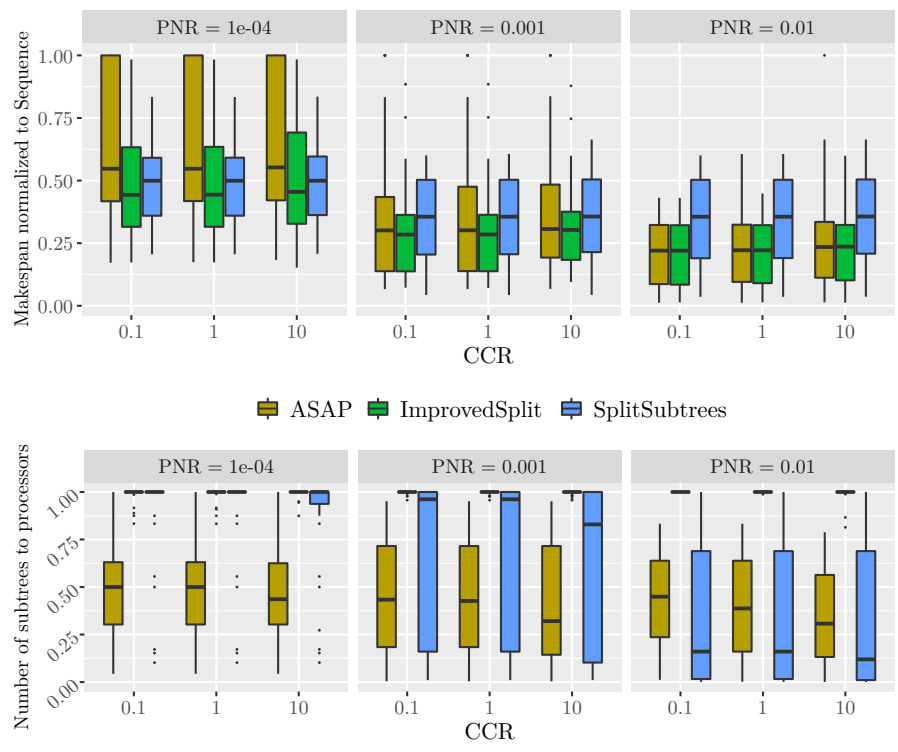

Figure 6: Makespan (top, normalized to Sequence) and number of generated subtrees (bottom) after Step 1 with different CCRs and PNRs.

Sequence, four times better on more than $50 \%$ of the cases. Increasing the number of processors generally allows us to reduce the makespan, except for SplitSubtrees. All heuristics behave better than SplitSubtrees for all CCR values. Also, note that ImprovedSplit always surpasses ASAP with few processors $(\mathrm{PNR}=1 e-04$ or 0.001$)$.

Figure ?? also presents the number of subtrees that are generated compared to the number of processors provided. Only ImprovedSplit takes fully advantage of processor resources in all cases. SplitSubtrees uses all processors only with few processors $(\mathrm{PNR}=1 e-04)$ and not with more processors because it only splits in two levels. For instance, for $\mathrm{PNR}=0.01$, SplitSubtrees uses $16 \%$ of the processors on more than $50 \%$ of the cases. ASAP uses much fewer processors than ImprovedSplit, using only half of the processors in around 50\% of the cases.

\subsection{Step 2: Fitting into memory}

At the end of Step 1, some subtrees may exceed the maximum available memory $M$ when we consider the strict memory scenario. As expected, there are less subtrees not fitting into memory when there are many processors, since subtrees are smaller, and also when using ImprovedSplit, since it generates more subtrees, and hence smaller subtrees. The subtrees that do not fit into memory are further decomposed with either FirstFit, LargestFirst or Immediately, so that all subtrees fit in memory at the end of this step. We may then have more subtrees than processors, and Step 3 will later merge subtrees if needed.

In order to assess the performance of the heuristics from Step 2, we execute them in the strict memory scenario both on the original tree (Sequence, i.e., no heuristic from Step 1 is used) and after running the heuristics from Step 1. We report the average ratio of number of subtrees to processors NtoP, and the average percentage of gain on execution time:

$$
\mathrm{ET}=100 \times\left(1-\frac{M S_{\infty}^{2}}{M S^{1}}\right)
$$




\begin{tabular}{cl|ll|ll|ll}
\hline \multirow{2}{*}{ PNR } & \multicolumn{2}{|c}{$1 \mathrm{e}-04$} & \multicolumn{2}{c}{0.001} & \multicolumn{2}{c}{0.01} \\
& & NtoP & ET & NtoP & ET & NtoP & ET \\
\hline \multirow{5}{*}{ FirstFit } & ASAP & 1.34 & $7 \%$ & 0.51 & $4 \%$ & 0.40 & $0 \%$ \\
& ImprovedSplit & 1.81 & $7 \%$ & 1.09 & $2 \%$ & 1.00 & $0 \%$ \\
& SplitSubtrees & 1.66 & $8 \%$ & 0.70 & $0 \%$ & 0.33 & $0 \%$ \\
& Sequence & 1.49 & $13 \%$ & 0.20 & $13 \%$ & 0.02 & $13 \%$ \\
\hline \multirow{5}{*}{ LargestFirst } & ASAP & 1.59 & $8 \%$ & 0.51 & $4 \%$ & 0.40 & $0 \%$ \\
& ImprovedSplit & 2.07 & $9 \%$ & 1.10 & $4 \%$ & 1.00 & $0 \%$ \\
& SplitSubtrees & 1.86 & $10 \%$ & 0.71 & $0 \%$ & 0.33 & $0 \%$ \\
& Sequence & 2.17 & $13 \%$ & 0.28 & $13 \%$ & 0.03 & $13 \%$ \\
\hline \multirow{5}{*}{ Immediately } & ASAP & 5.97 & $9 \%$ & 0.90 & $6 \%$ & 0.41 & $2 \%$ \\
& ImprovedSplit & 5.61 & $5 \%$ & 1.38 & $1 \%$ & 1.00 & $0 \%$ \\
& SplitSubtrees & 5.13 & $9 \%$ & 0.97 & $4 \%$ & 0.33 & $0 \%$ \\
& Sequence & 6.24 & $18 \%$ & 0.90 & $18 \%$ & 0.09 & $18 \%$ \\
\hline
\end{tabular}

Table 1: After Step 2, NtoP is the ratio of number of subtrees to processors, and $\mathrm{ET}$ is the gain on execution time. $\mathrm{CCR}=1$.

where $M S_{\infty}^{2}$ is the makespan after Step 2 with an infinite number of processors (since splitting subtrees in Step 2 may generate more subtrees than available processors), and $M S^{1}$ is the makespan after Step 1. If ET is positive, it means that the new makespan is better, and it is feasible only if NtoP is smaller than or equal to 1 .

Table ?? presents all results, for the three heuristics of Step 2 (FirstFit, LargestFirst and Immediately) and the four possibilities for Step 1, for CCR=1. Overall, FirstFit generates the smallest amount of subtrees, hence it is more likely that this heuristic will succeed to map all subtrees to processors while fitting in memory. LargestFirst has close results in terms of number of subtrees, and it is interesting to see that it can reduce the makespan even more than FirstFit. Immediately generates much more subtrees, and, in some cases, it may be able to further decrease the makespan.

Results for other values of CCR are available in the companion research report [?]. They lead to the same conclusions, even though there is less gain in terms of makespan (and sometimes even an increase in makespan) for $\mathrm{CCR}=10$, since creating additional subtrees to fit into memory may generate expensive communications.

In the following, we always apply LargestFirst for Step 2 in the strict scenario. Indeed, LargestFirst obtains convincing results with a reasonable number of subtrees and interesting improvement in execution time. We refer readers interested in the results with FirstFit and Immediately to the companion research report [?].

\subsection{Step 3: Reaching an acceptable number of subtrees}

In this section, we examine the performance of Merge and SplitAgain, which are designed for reducing the number of subtrees so that we have enough processors, or for further optimizing the makespan if there are some remaining processors. As seen in Table ??, ImprovedSplit is the heuristic generating the most subtrees when combined with LargestFirst, and hence it requires to merge some subtrees to obtain a feasible solution. The other heuristics leave many processors idle when there are many processors $(\mathrm{PNR}=0.001$ or $\mathrm{PNR}=0.01)$, and we may be able to further improve the makespan by using SplitAgain in these cases.

Figure ?? shows the performance of SplitAgain or Merge. It plots the ratio of makespan after Step 3 to the execution time that was achieved at the end of Step 2 (with an infinite number of processors), using LargestFirst during Step 2 (strict memory scenario), with $\mathrm{CCR}=0.1$. Each tile in the figure represents a testing case. Green tiles mean that the ratio is smaller than one, i.e., the makespan was improved, while red tiles represent ratios greater than one. Finally, grey

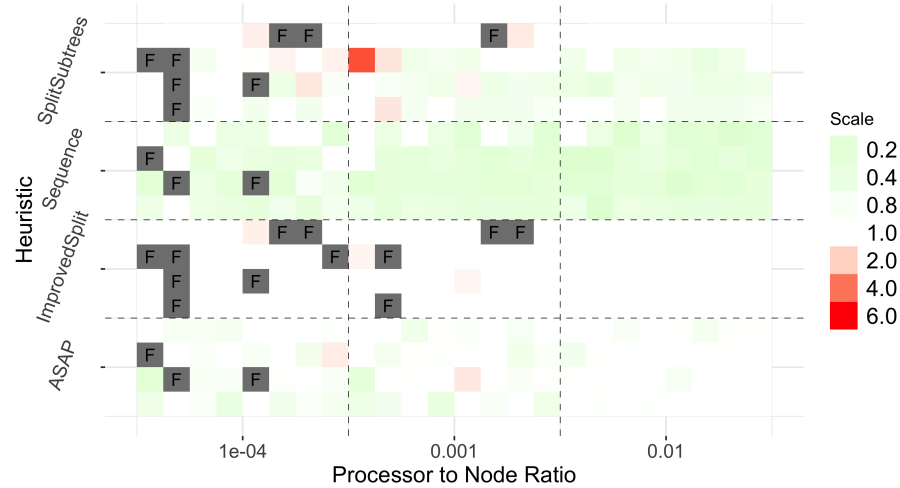

Figure 7: Ratio of makespan after Step 3 to execution time after Step 2 (with infinite number of processors), using LargestFirst at Step 2. F represents a failure. $\mathrm{CCR}=0.1$.
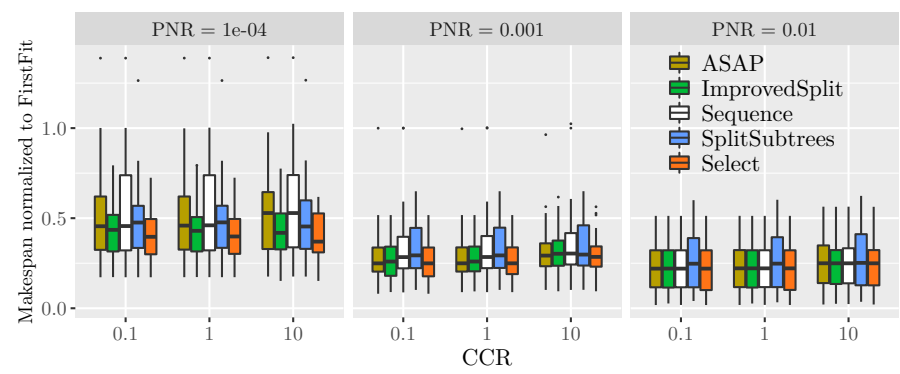

Figure 8: Final makespan (using Step 1 heuristics followed by LargestFirst and SplitAgain/Merge) normalized to FirstFit.

tiles with $\mathrm{F}$ represent a failure, i.e., we were not able to obtain a solution with less subtrees than processors. This may happen since there is a strict memory constraint and a limited number of processors, and it might not be possible to execute a given tree on the platform that we consider, even without trying to optimize the makespan. As expected, the less processors, the more failures we have. Since Sequence did nothing at Step 1, it starts from a sequential execution of the tree and hence it obtains important gains in makespan after using SplitAgain in Step 3. SplitAgain also allows us to improve the makespan with ASAP and SplitSubtrees. As noted before, ImprovedSplit usually generates more subtrees than processors after Step 2, and hence we must use Merge to obtain a feasible solution, as well as for other heuristics when there are few processors $(\mathrm{PNR}=1 \mathrm{e}-04)$. We observe some failures in these cases, in particular when using ImprovedSplit, while ASAP and Sequence succeed in most cases. Overall, the failure rate after Step 3 is $7.26 \%$.

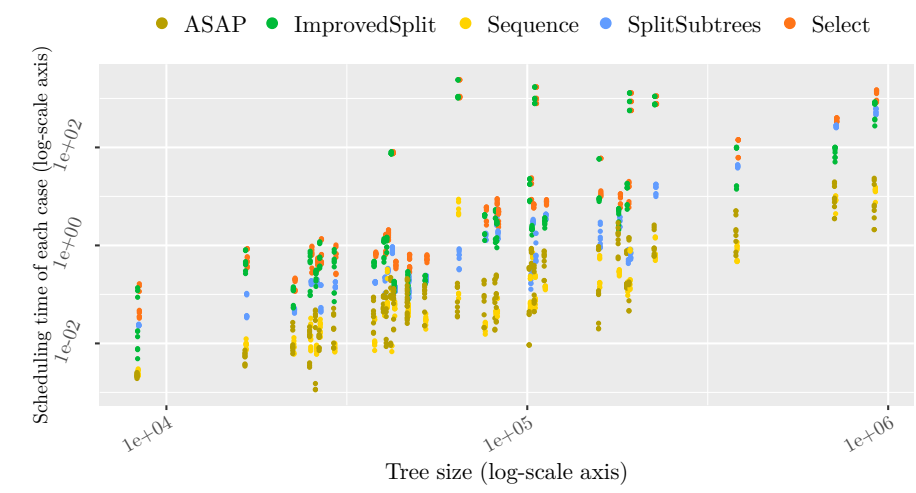

Figure 9: Scheduling time in minutes of different allocation policies, all followed by LargestFirst and SplitAgain or Merge. 
Still in the strict memory scenario, we finally compare the makespan of all our heuristics to FirstFit, since it is a simple adaptation from [?]. Indeed, FirstFit is likely to give a feasible solution in most cases, since it consumes least processors, as shown in Table ??. Furthermore, we consider the heuristic Select, which runs all possible heuristics at Step 1 (followed by LargestFirst, and then SplitAgain or Merge), and keeps the best solution for each input tree. This allows us to analyze whether there is at least one heuristic that outperforms others in all situations. Figure ?? presents the final makespan obtained after all three steps, excluding cases on which no solution was found. Recall that failure rates can be found in Figure ??. We first note that all proposed heuristics allow us to largely reduce the makespan compared to FirstFit, and they give very similar results, especially for large PNRs. Overall, ImprovedSplit is the best heuristic when there are a few processors $(\mathrm{PNR}=1 \mathrm{e}-04)$, but other heuristics outperform it in several cases, since Select is even better achieving a makespan 2.5 times faster than the reference FirstFit. With more processors, ASAP is slightly better, in particular for $\mathrm{PNR}=0.001$. Skipping Step 1 (Sequence) gives reasonable results as soon as there are many processors $(\mathrm{PNR}=0.01$, or even $\mathrm{PNR}=0.001$ ), which shows that the heuristics from Step 3 (SplitAgain and Merge) are very efficient in these cases (in particular SplitAgain). With $\mathrm{PNR}=0.01$, all heuristics achieve a makespan four times smaller than the reference, in average. Finally, note that we may get a makespan worse than the reference on some cases, in particular with Sequence and SplitSubtrees (1.39 or 1.26 times worse than the makespan from FirstFit), but these outlier cases are avoided by using Select.

Of course, Select is always the best pick, but it may come at the price of a higher scheduling time, since it implies to run all four variants. We report the execution times in Figure ??. To ease the reading, we plot the scheduling time (in minutes) and number of nodes in the tree on logarithmic scale axes. ASAP and Sequence are the fastest heuristics, and it is interesting to note that ASAP can sometimes be even faster than Sequence, even though Sequence does not do anything in Step 1: the tree obtained at the end of Step 1 with ASAP has then a faster scheduling time for Steps 2 and 3 than starting from the original tree. As expected, ImprovedSplit takes more time than SplitSubtrees, since it refines the solution from SplitSubtrees to cut in several levels. It has to be noted that very long scheduling times (above 10 minutes) only happen for very large trees (above 100,000 nodes), except for a few extreme cases. Overall, running Select is only slightly longer than ImprovedSplit, since the scheduling times of all other heuristics are small in comparison to the one of ImprovedSplit. Finally, note that different processor to node ratios (PNR) only slightly impact the scheduling time (see [?] for detailed results).

To summarize, we recommend using Select, unless the scheduling time is very important or the tree is very large, in which cases ASAP is a good option for Step 1 (efficient makespan obtained with a fast execution of the heuristic).

Finally, we present results in the loose memory scenario, where the memory bound for each processor is set to MinMemory, hence there is no memory constraint. In this case, we only consider the use of Step 1 directly followed by

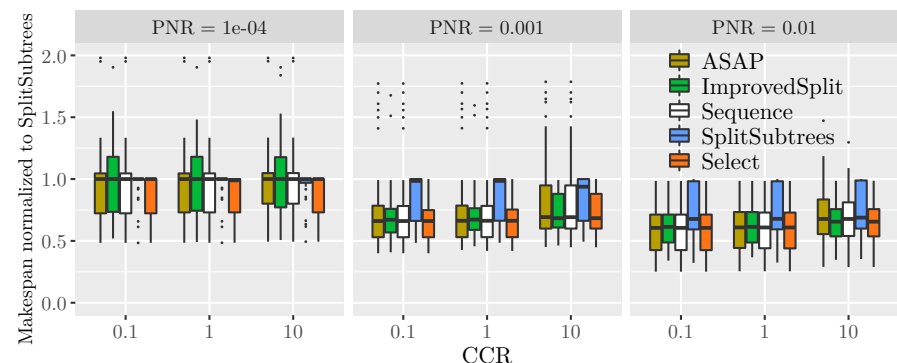

Figure 10: Final makespan of all Step 1 heuristics followed by SplitAgain, normalized to SplitSubtrees (without SplitAgain) in the loose memory scenario.

Step 3. The reference heuristic becomes SplitSubtrees, which was directly adapted from ideas from [?], resulting in a twolevel split of the tree.

Figure ?? reports the final makespan, after applying one heuristic of Step 1 followed by SplitAgain. Indeed, at the end of Step 1, there are always less subtrees than processors. With few processors $(\mathrm{PNR}=1 \mathrm{e}-04)$, there is little room for improvement over the two-level partitioning of the tree. However, when the number of processors increase, SplitAgain achieves good results in using available processors to reduce the makespan, even without going through a heuristic from Step 1 (Sequence variant). Using only SplitAgain on the original tree is a good option in this case. Note that again, apart from SplitSubtrees, all heuristics give close results.

\section{Conclusion}

We have studied a tree partitioning problem, targeting at a multiprocessor computing system in which each processor has its own local memory. The tree represents dependencies between tasks, and it can be partitioned into subtrees, where each subtree is executed by a distinct processor. The goal is to minimize the time required to compute the whole tree (makespan), given some memory constraints: the minimum memory requirement of traversing each subtree should not be more than the local memory capacity. We have proved that the problem above, MinMakespan, is NP-complete, and we have designed several heuristics to tackle it. We propose a three-step approach: (i) minimize the makespan; (ii) fit into memory if needed; and (iii) make sure that we have less subtrees than processors, and use as many processors as required to further minimize the makespan.

Extensive simulations demonstrate the efficiency of these heuristics and provide guidelines about the heuristics that should be used. Without memory constraint, the heuristic from Step 3, SplitAgain, is efficiently splitting the tree to minimize the makespan, and achieves results 1.5 times better than the reference heuristic, SplitSubtrees, when there are many processors available (processor to node ratio $\mathrm{PNR} \geq 0.001$ ). When there are memory constraints, one must make sure that each subtree fits into memory, and the reference heuristic is FirstFit, which partitions the tree for memory. In this case, using the best combination of a heuristic of Step 1, a heuristic to fit into memory, and finally SplitSubtrees or Merge, allows us to drastically improve the makespan (two to four times better, depending on the processor to node ratio). The use of ASAP in Step 1 may be selected for a smaller scheduling 
time, since ImprovedSplit may lead to a smaller makespan, but at the price of a longer scheduling time.

Building upon these promising results, an interesting direction for future work would be to consider partitions that do not necessarily rely on subtrees, but where a single processor may handle several subtrees. Also, we plan to extend this work to general directed acyclic graphs of task, while we have restricted the approach to trees so far. Adapting existing graph partitioners to account for memory constraints (as done in [?]) and distributed processing is also a promising research direction.

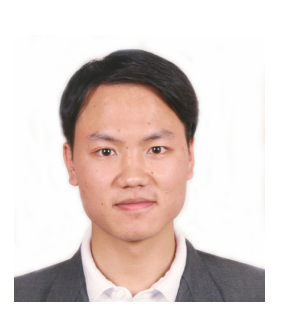

Changjiang Gou is a PhD candidate in Computer Sciences at the LIP laboratory of École Normale Supérieure de Lyon (ENS Lyon), France, and East China Normal University (ECNU), Shanghai, China. He works under the supervision of Anne Benoit, Loris Marchal and MingSong Chen.

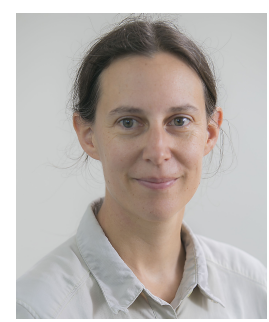

Anne Benoit received the $\mathrm{PhD}$ degree from Institut National Polytechnique de Grenoble in 2003, and the Habilitation à Diriger des Recherches (HDR) from ENS Lyon in 2009. She is currently an associate professor in the Computer Science Laboratory LIP at ENS Lyon, France. She is Associate Editor (in Chief) of Parco, and Associate Editor of IEEE TPDS and JPDC. She is a senior member of the IEEE, and she has been elected a Junior 2009. Member of Institut Universitaire de France in

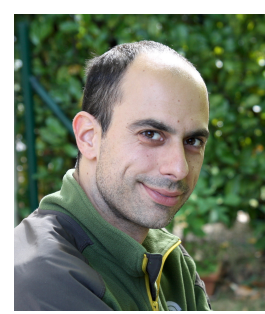

Loris Marchal graduated in Computer Sciences and received his $\mathrm{PhD}$ from École Normale Supérieure de Lyon (ENS Lyon, France) in 2006. He is now a CNRS researcher at the LIP laboratory of ENS Lyon. His research interests include parallel computing and scheduling. 